\title{
Adsorption of comb copolymers on weakly attractive solid surfaces
}

\author{
A. Striolo ${ }^{a}$ \\ Department of Chemical Engineering, Vanderbilt University, Nashville, Tennessee 37235
}

\author{
A. Jayaraman, J. Genzer, and C. K. Hall \\ Department of Chemical and Biomolecular Engineering, North Carolina State University, Raleigh, North \\ Carolina 27605
}

(Received 9 March 2005; accepted 12 June 2005; published online 17 August 2005)

\begin{abstract}
In this work continuum and lattice Monte Carlo simulation methods are used to study the adsorption of linear and comb polymers on flat surfaces. Selected polymer segments, located at the tips of the side chains in comb polymers or equally spaced along the linear polymers, are attracted to each other and to the surface via square-well potentials. The rest of the polymer segments are modeled as tangent hard spheres in the continuum model and as self-avoiding random walks in the lattice model. Results are presented in terms of segment-density profiles, distribution functions, and radii of gyration of the adsorbed polymers. At infinite dilution the presence of short side chains promotes the adsorption of polymers favoring both a decrease in the depletion-layer thickness and a spreading of the polymer molecule on the surface. The presence of long side chains favors the adsorption of polymers on the surface, but does not permit the spreading of the polymers. At finite concentration linear polymers and comb polymers with long side chains readily adsorb on the solid surface, while comb polymers with short side chains are unlikely to adsorb. The simple models of comb copolymers with short side chains used here show properties similar to those of associating polymers and of globular proteins in aqueous solutions, and can be used as a first approximation to investigate the mechanism of adsorption of proteins onto hydrophobic surfaces. (C) 2005 American Institute of Physics. [DOI: 10.1063/1.1993557]
\end{abstract}

\section{INTRODUCTION}

Polymers adsorbed onto solid surfaces are frequently encountered in nature and in technological applications. Polymer adsorption is used to stabilize or flocculate colloidal suspensions (paints, coatings, inks, etc.), ${ }^{1,2}$ to improve the performance of membranes for protein fractionation, ${ }^{3,4}$ to increase the circulation half-life of liposomes in the blood stream, ${ }^{5}$ and to engineer biocompatible materials. ${ }^{6}$ The adsorption of biopolymers (e.g., proteins) at solid surfaces is of relevance in a wide range of applications including industrial biocatalysis and stabilization of therapeutic proteins. ${ }^{7,8}$

At dilute conditions the behavior of a polymer chain near a surface is determined primarily by polymer-surface interactions and solvent quality. ${ }^{9}$ When polymer-surface interactions are favorable for adsorption, the conformational statistics of polymer chains in the vicinity of the solid surface is determined by a competition between the energetic advantage of segmental adsorption and the associated entropic penalty. The entropic penalty arises because adsorption reduces both the translational freedom and the number of available conformations for the polymer chain. Due to the presence of hydrophilic and hydrophobic residues, the adsorption of proteins and associating polymers on a surface is more complicated than the adsorption of homopolymers. When proteins are dissolved in a bulk aqueous solution the

\footnotetext{
${ }^{a}$ Present address: University of Oklahoma, School of Chemical Biological and Materials Engineering, Norman OK 73019. Electronic mail: astriolo@ou.edu
}

hydrophobic residues are preferentially located in the interior of the macromolecules while hydrophilic residues are exposed to the aqueous environment. When adsorbed on a surface, proteins often undergo conformational changes in an attempt to lower the global free energy. ${ }^{10}$

Mean-field theories are useful for understanding polymer adsorption. Fang and Szleifer ${ }^{11,12}$ developed a molecular theory to study the thermodynamics and the kinetics of the adsorption of proteins of different sizes and charges on a solid surface. Carignano and Szleifer ${ }^{13}$ showed that by grafting charged polymers on an attractive surface it is possible to prevent primary protein adsorption (the adsorption of proteins directly onto the attractive solid surface), and to promote secondary adsorption (the adsorption of proteins onto the layers of grafted polymers, but not onto the solid surface). Scanning-probe microscopy has been used to visualize macromolecules adsorbed onto solid surfaces. ${ }^{14}$ For example, Bauerle et al. ${ }^{15}$ and Prokhorova et al. ${ }^{16}$ observed monolayers with long-range order when oligo(thiophenes) or comblike polymers adsorb on graphite, while Sheiko et al. ${ }^{17}$ reported first-order rod-globule phase transitions in adsorbed polymer films. Molecular simulations provide a bridge between mean-field theories and experimental data. ${ }^{18}$ For example, Zajac and Chakrabarti ${ }^{19}$ and Striolo and Prausnitz ${ }^{20}$ reported Monte Carlo simulations for the adsorption of linear homopolymers, branched homopolymers, and copolymers on solid surfaces.

In this work we use Monte Carlo simulations to study the adsorption of linear and comb polymers on solid 
surfaces. We use a continuum model to study the adsorption of polymers from infinitely dilute solutions. We complement these studies with a lattice model to investigate the adsorption of polymers from finite-concentration solutions. Polymer-segment-segment and segment-surface interactions are modeled using hard-sphere potentials. Selected polymer segments, equally spaced along the linear polymers or located at the tips of the side chains in comb polymers, are attracted to each other and to the surface; these are modeled using square-well potentials. Polymers are considered adsorbed when the density of polymer segments near the surface is larger than that in the bulk. In order to investigate the characteristics of the polymer architecture that lead to maximum adsorption, we vary the lengths of the side chains [zero (linear polymer), one, three, five, eight, and ten segments] and the lengths of the backbone $(69,99$, and 129 segments) in the different simulation runs. To understand why one certain polymer architecture favors adsorption, we compare the conformations of adsorbed polymers to those of polymers in bulk solutions.

We chose the comb polymer architecture to crudely represent proteins. Secondary and tertiary structures of globular proteins in aqueous solutions are primarily due to hydrogen bonds, hydrophobic interactions between side groups of the amino acids (for example, phenylalanine and tryptophan contain aromatic rings), disulfide bridges between cysteine residues, and salt bridges between charged side groups. ${ }^{21}$ The strongly associating sites are located at a short distance from the protein backbone. We mimic the protein architecture by using comb polymers with short side chains where the tips of the side chains attract each other. This representation is crude, and certainly does not allow us to model secondary protein structures such as $\alpha$ helices or $\beta$ sheets, but it is sufficient to understand if the architecture of proteins has any impact on their thermodynamic behavior (e.g., adsorption on hydrophobic surfaces).

At infinite dilution, our results show that linear polymers do not adsorb readily on a surface because of large entropic penalties. Comb polymers with short side chains readily adsorb on a solid surface and undergo conformational changes. These polymers assume globular conformations in the bulk and spread upon adsorption. Comb polymers with long side chains adsorb on a surface but maintain a globular conformation. Interestingly, the behavior of comb polymers with short side chains resembles that of proteins which are globular in bulk aqueous solutions and spread on top of hydrophobic surfaces. At finite concentration our results show that linear polymer and comb polymers with long side chains readily adsorb on the solid surface. Linear polymers adsorb and spread on the surface, and entangle with each other. Adsorbed comb polymers do not entangle with each other, but maintain globular conformations. The typical conformation of adsorbed comb polymers is characterized by few attractive segments in contact with the surface, and others at some short distance from it.

The remainder of this paper is organized as follows: in Sec. II we describe the simulation details for the infinitely dilute continuum model and the finite-concentration lattice model. In Sec. III we discuss the results obtained from the

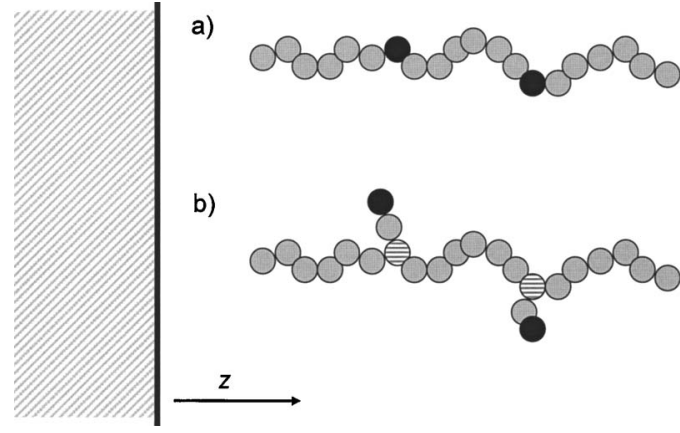

FIG. 1. Schematic representation of one linear polymer (a) and one comb polymer (b). The black segments interact with each other and with the surface via attractive square-well potentials [Eqs. (1) and (2) in the text]. The gray segments are modeled as hard spheres. The striped segments in the comb polymer (b) represent the branching segments and are treated as hard spheres.

infinitely dilute continuum simulations and the finiteconcentration lattice simulations. In Sec. IV we provide a brief summary of our conclusions.

\section{MODEL AND SIMULATION DETAILS}

\section{A. Infinitely dilute continuum model}

We use continuum Monte Carlo simulations to study the adsorption of comb and linear polymers on a flat solid surface when the system is at infinitely dilute conditions. We treat comb and linear polymers as sequences of freely jointed tangent spheres. We use the ensemble-growth algorithm, described below, to grow an equilibrated ensemble of systems composed of one polymer near the attractive surface. In Fig. 1 we show a schematic representation of the polymer architecture of one linear polymer [Fig. 1(a)] and one comb polymer [Fig. 1(b)]. The shaded region on the left side of the figure represents the attractive solid surface modeled as a flat wall located at $z=0$. The $z$ coordinate indicates the distance from the attractive surface. Polymer segments are allowed only in the region of positive $z$. In this work all distances are measured in terms of diameter of polymer segment, $\sigma$, which is considered as the unit length. The linear polymer shown in Fig. 1(a) is composed of 20 segments. Two of the segments are attractive and they are separated from each other by six nonattractive segments. The comb polymer shown in Fig. 1(b) is composed of a backbone of length 20 segments and two side chains, each of length two segments, separated from each other by six nonattractive segments. The length of the side chains does not account for the branching segment [striped bead in Fig. 1(b)] which is part of the backbone. The attractive segments (black beads) are either equally spaced along the linear polymer [Fig. 1(a)] or located at the tips of the side chains [Fig. 1(b)]; these are attracted to each other and to the solid surface. The other segments (gray and striped beads) are nonattractive. The conformational details (number of segments in the backbone, $N_{\text {backbone }}$, number of segments in each side chain, $N_{\text {side chain, }}$ and total number of segments in the polymer, $N_{\text {total }}$ ) for all the polymers considered are given in Table I. All comb and linear polymers contain nine attractive segments at the tips of the nine side chains (except for linear polymers in which the attractive 
TABLE I. Structural characteristics of the model polymers simulated in this work. $N_{\text {backbone }}$ is the number of segments in the backbone, $N_{\text {side chain }}$ is the number of segments in the side chain (all of the same length within a single polymer), and $N_{\text {total }}$ is the total number of segments in the polymer. All polymers except the linear ones have nine side chains. In all polymers the total number of segments attracted to each other and to the surface is 9 .

\begin{tabular}{lccc}
\hline \hline Polymer & $N_{\text {backbone }}$ & $N_{\text {side chain }}$ & $N_{\text {total }}$ \\
\hline Linear_69 & 69 & $\ldots$ & 69 \\
Linear_99 & 99 & $\ldots$ & 99 \\
Linear_129 & 129 & $\ldots$ & 129 \\
Comb1_69 & 69 & 1 & 78 \\
Comb1_99 & 99 & 1 & 108 \\
Comb1_129 & 129 & 1 & 138 \\
Comb3_69 & 69 & 3 & 96 \\
Comb3_99 & 99 & 3 & 126 \\
Comb3_129 & 129 & 3 & 153 \\
Comb5_69 & 69 & 5 & 114 \\
Comb5_99 & 99 & 5 & 144 \\
Comb5_129 & 129 & 5 & 174 \\
Comb8_99 & 99 & 8 & 171 \\
Comb8_129 & 129 & 8 & 201 \\
Comb10_69 & 69 & 10 & 159 \\
Comb10_99 & 99 & 10 & 189 \\
Comb10_129 & 129 & 10 & 219 \\
\hline \hline
\end{tabular}

segments are embedded in the backbone). We consider polymers with backbones of lengths 69, 99, and 129 segments $\left(N_{\text {backbone }}\right)$ and side chains of lengths one, three, five, eight, and ten segments $\left(N_{\text {side chain }}\right)$. For the three values of $N_{\text {backbone }}$ the corresponding lengths on the backbone that separates two consecutive side chains are 6, 9, and 12 segments, respectively.

To describe segment-segment interactions we choose simple potentials. We recognize that, as a first approximation, specific interactions between the side groups of the amino acids are primarily responsible for the structure of proteins. Thus we assign attractive square-well potentials to describe interactions between attractive segments, and hardsphere potentials to describe all other interactions. Algebraically, for effective interactions between segments $i$ and $j$ we use square-well potentials $\phi_{i j}$,

$$
\phi_{i j}=\left\{\begin{array}{lll}
\infty & & 0<d_{i j}<1 \\
-\varepsilon & \text { for } \quad & 1 \leqslant d_{i j} \leqslant 1.5 \\
0 & & 1.5<d .
\end{array}\right.
$$

In Eq. (1) $d$ is the center-to-center distance between polymer segments and $\varepsilon$ is the well depth. The well depth $\varepsilon$ is nonzero only for the attractive polymer segments. All other interactions are hard-sphere interactions. The strength of the specific attractions depends, among other things, on the type of amino acid. For the sake of simplicity we conduct simulations for $\varepsilon$ ranging between 2 and $4 k T$.

The segment-surface interactions are represented by square-well potentials $\theta$,

$$
\theta_{i}=\left\{\begin{array}{lll}
\infty & & 0<z_{i}<0.5 \\
-\xi & \text { for } \quad & 0.5 \leqslant z_{i} \leqslant \lambda \\
0 & & \lambda<z .
\end{array}\right.
$$

In Eq. (2), $z$ represents the distance of the polymer segment $i$ from the attractive wall, $\xi$ represents the depth of the segment-surface attractive interaction, and $\lambda$ represents the width of the attractive well. The segment-surface well depth $\xi$ is attractive only for those polymer segments that attract each other via the square-well potential of Eq. (1). For all the other segments, the surface is effectively a hard wall. We consider three values for $\lambda(0.75,1$, or 1.5$)$. As $\lambda$ increases, the segment-surface interaction becomes longer ranged.

To obtain an equilibrated ensemble of systems each composed of one polymer near the surface we implement the ensemble-growth algorithm. ${ }^{22-24}$ The algorithm builds simultaneously, step by step, an ensemble of $M_{c}$ systems, each composed of one polymer near the surface. The first polymer segment of each of the $M_{c}$ polymer-surface systems is inserted randomly at a distance between 0.5 and 30 from the attractive surface. For each of the $M_{c}$ members of the ensemble five possible positions for the second polymer segment are randomly chosen around the first segment. Out of the total $5 M_{c}$ possibilities, $M_{c}$ polymer-surface systems are randomly chosen according to Boltzmann probability. The systems are stored as the new members of the ensemble. The remaining $4 M_{c}$ replicas are erased from the memory. This procedure guarantees that energetically favorable polymersurface systems are more likely to be chosen as members of the new ensemble than energetically unfavorable systems. The correct implementation of the algorithm requires some less-favorable polymer-surface systems to be statistically present in the ensemble at all times. This is achieved only for large values of $M_{c}$. In the next step the third segments are attached to the second segment using the same procedure that is adopted to attach the second segments to the first segment. The procedure is repeated until $M_{c}$ polymer-surface replicas of fully grown polymers are obtained. The same algorithm is also used to obtain bulk polymer systems, where we neglect the presence of the surface for the purpose of comparison to our polymer-surface systems. Further details on the algorithm can be found in Ref. 24. For both polymersurface systems and bulk polymer systems the quantities of interest (segment-density profiles, axial distribution functions, and radii of gyration) are computed and averaged over the $M_{c}$ polymer-surface systems that comprise the final ensemble. For polymer-surface systems, each ensemble contains $M_{c}=40000$ replicas and the results are averaged over at least 50 independent ensembles. When the surface is strongly attractive, the simulation data are rather noisy; up to 150 independent ensembles are required to obtain reliable results. For bulk polymer systems, each ensemble contains $M_{c}$ $=30000$ replicas and the results are averaged over at least three independent ensembles. The independent ensembles are obtained by repeating the simulations with a different seed for the random number generator. To assess the reproducibility of our results we compute the average of the different runs, the standard deviation from the average, and the percentage of the standard deviation with respect to the av- 
erage. This percentage of the standard deviation with respect to the average is referred to as "percentage error" in our results.

\section{B. Finite-concentration lattice model}

Protein solutions are seldom at infinitely dilute concentrations. However, the ensemble-growth algorithm requires too much memory for the investigation of systems at finite concentration. We employ a lattice model to study adsorption of polymer systems at finite concentration. Lattice Monte Carlo simulation is computationally fast and yet provides a good description of the large scale conformational features of polymer chains. We perform the simulations on a cubic lattice in the canonical $(N V T)$ ensemble, i.e., the total number of segments in the simulation box $(N)$, the volume of the simulation box $(V)$, and the temperature of the system $(T)$ are maintained constant. The simulation box has periodic boundary conditions in the $x$ and $y$ directions. In the $z$ direction there is an impenetrable surface at $z=h+1$ and an adsorbing surface at $z=0$, where $h$ is the height of the simulation box. The simulation box dimensions in the $x, y$, and $z$ directions are 30,30 , and 50, respectively. The polymers are modeled as self-avoiding walks on the cubic lattice. Polymer segments located at the tips of side chains in comb polymers or equally spaced in linear polymers are attracted to each other and to the surface by a square-well attraction of $2 k T$. This value of the potential is strong enough to induce the polymers to adsorb at the attractive surface, yet weak enough to allow them to desorb. The rest of the polymer segments interact with each other and with the surface via athermal potentials $(\varepsilon=0 k T)$. The polymer-segment-segment interaction potential is short ranged and only affects nonbonded nearest neighbors. The segment-surface interaction potential is attractive only when the polymer segments occupy lattice sites on the plane $(z=1)$ adjacent to the adsorbing surface $(z=0)$. We vary the lengths of the side chains [zero (linear polymer), one, three, five, eight, and ten segments] while we maintain $N_{\text {backbone }}=99$ in the different simulation runs.

The initial configuration of the copolymer chains is obtained by first growing the backbone and then adding the side chains to the branch points. The backbone is grown by first placing the head segments of the chains at random locations on the lattice. The second segments are placed on sites adjacent to the head segment on each chain, one by one. The third segments are placed on sites next to the second segment, one at a time, and this procedure is repeated until the backbones are grown to the desired length. During this process, if there is no vacant lattice site for adding a segment to one end of one chain, the segment is added to the other end of the chain. If adding the segment to either end fails (because there is no vacant site), then the chains are moved in the box (using translation, kink jump, and crankshaft moves) until a vacancy next to the chain in question is created. Once the backbone is grown completely, the side chains are added to the backbones in a similar fashion. Attempted Monte Carlo moves comprise the usual assortment of local chain moves (end moves, kink jump, and crankshaft moves $)^{25}$ in addition to translation moves. The type of move,

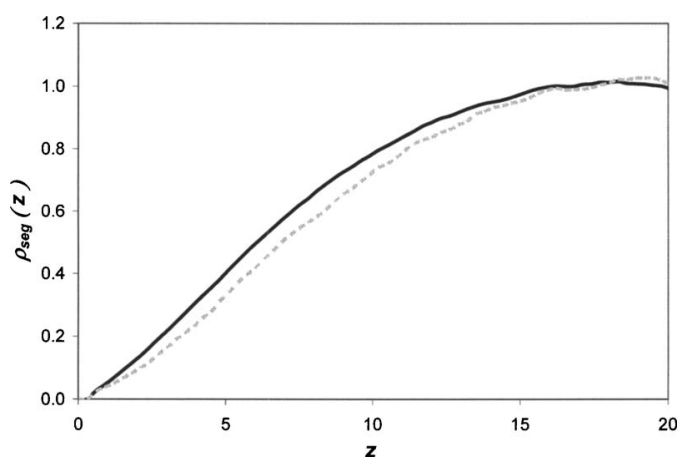

FIG. 2. Segment density $\rho(z)$ as a function of the separation from the attractive surface, $z$, for linear polymers (black solid line) and comb polymers with ten-segment-long side chains (gray dashed line) when $\xi=2 k T$ and $\lambda$ $=0.75$. For both polymers $N_{\text {backbone }}=99$. The percentage error in these data is below $5 \%$ except at close separations from the wall $(z<2)$ where the percentage error can be as high as $50 \%$ due to the small absolute values computed.

chain, and segment number along the chain is chosen randomly. In one Monte Carlo (MC) step we make $N_{\text {polymers }}$ $\times N_{\text {total }}$ moves, where $N_{\text {polymers }}$ is the number of polymers in the simulation box and $N_{\text {total }}$ is the number of segments in each polymer. The moves are accepted or rejected according to the Metropolis algorithm. ${ }^{26}$ The simulation comprises three stages: initialization, equilibration, and production. In the initialization stage we let the system go through 100000 MC steps, moving the chains in the simulation box while polymer-segment-segment and segment-surface attractive interactions are switched off. This stage is required to avoid any bias due to the nature of the initial configuration. In the equilibration stage we let the system go through $2 \times 10^{6} \mathrm{MC}$ steps while the polymer-segment-segment and polymersegment-surface attractive interactions are switched on. In the production stage the data for the quantities of interest (e.g., segment-density profiles) are collected.

\section{RESULTS AND DISCUSSION}

\section{A. Infinitely dilute continuum model \\ 1. Effect of varying $\xi$ and $\lambda$}

In Fig. 2 we report the segment-density profile as a function of the distance $z$ from the adsorbing surface for 99segment-long linear polymers (black solid line), and comb polymers with 99 -segment-long backbones and 10-segmentlong side chains (gray dashed line) when $\xi=2 k T$ and $\lambda$ $=0.75$. The segment-density profile at a distance $z$ from the surface is obtained as the ratio of the average density of polymer segments found at distance $z$ and the average density of polymer segments in the bulk (equal to the average polymer-segment density obtained at distances between 18 and 20 from the surface). The segment-density profiles of the comb polymers with side chains of lengths one, three, and five segments are not shown in Fig. 2 for clarity. Our results indicate that the segment density near the surface is less than that in the bulk and that it approaches zero as $z \rightarrow 0$. The region near a solid surface where the segment density is less than that in the bulk is known as the depletion layer. ${ }^{27,28}$ It is possible, even at inifinitely dilute concentrations, to define the depletion-layer thickness, $\delta,{ }^{20,29}$ according to 
TABLE II. Width of the depletion layer $\delta$ and parameter $\Theta$ [see Eqs. (3) and (4) for definition] obtained from the simulation of polymer adsorption in the infinitely dilute continuum model. The acronyms used to identify the polymer architectures correspond to those in Table I.

\begin{tabular}{|c|c|c|c|c|c|c|}
\hline \multirow[b]{2}{*}{ Polymer } & \multicolumn{2}{|c|}{$\lambda=0.75 ; \xi=2 k T$} & \multicolumn{2}{|c|}{$\lambda=1.00 ; \xi=2 k T$} & \multicolumn{2}{|c|}{$\lambda=1.50 ; \quad \xi=2 k T$} \\
\hline & $\delta$ & $\Theta$ & $\delta$ & $\Theta$ & $\delta$ & $\Theta$ \\
\hline Linear_99 & 6.71 & -40.99 & 6.34 & -37.01 & 4.79 & -15.12 \\
\hline Comb1_99 & 6.56 & -38.31 & & & 3.45 & 3.50 \\
\hline Comb3_99 & 6.56 & -35.64 & & & 1.92 & 24.99 \\
\hline Comb5_99 & 7.14 & -40.49 & 6.26 & -29.54 & 3.27 & 8.34 \\
\hline Comb8_99 & & & 6.65 & -31.56 & 3.66 & 5.51 \\
\hline \multirow[t]{3}{*}{ Comb10_99 } & 7.49 & -38.61 & & & 4.05 & 1.85 \\
\hline & \multicolumn{2}{|c|}{$\lambda=1.00 ; \xi=3 k T$} & \multicolumn{2}{|c|}{$\lambda=1.00 ; \xi=4 k T$} & & \\
\hline & $\delta$ & $\Theta$ & $\delta$ & $\Theta$ & & \\
\hline Linear_99 & 4.85 & -16.10 & -4.37 & 109.77 & & \\
\hline Comb1_99 & 2.30 & 19.19 & & & & \\
\hline Comb3_99 & -0.77 & 60.00 & -41.64 & 612.08 & & \\
\hline Comb5_99 & 0.11 & 48.65 & & & & \\
\hline Comb8_99 & 1.47 & 32.10 & & & & \\
\hline \multirow[t]{3}{*}{ Comb10_99 } & 2.17 & 24.11 & -34.01 & 457.89 & & \\
\hline & \multicolumn{2}{|c|}{$\lambda=1.00 ; \xi=3 k T$} & & & & \\
\hline & $\delta$ & $\Theta$ & & & & \\
\hline Linear_69 & 2.47 & 7.03 & & & & \\
\hline Comb1_69 & -1.71 & 78.98 & & & & \\
\hline Comb3_69 & -7.54 & 171.38 & & & & \\
\hline Comb5_69 & -7.14 & 160.57 & & & & \\
\hline Comb10_69 & -2.23 & 81.20 & & & & \\
\hline Linear_129 & 6.22 & -21.88 & & & & \\
\hline Comb1_129 & 4.89 & -5.65 & & & & \\
\hline Comb3_129 & 3.74 & 8.52 & & & & \\
\hline Comb5_129 & 2.88 & 18.67 & & & & \\
\hline Comb8_129 & 3.76 & 10.34 & & & & \\
\hline Comb10_129 & 4.36 & 4.96 & & & & \\
\hline
\end{tabular}

$$
\delta=\int_{0}^{\infty}\left(1-\frac{\rho_{\text {seg }}(z)}{\rho_{\text {seg }}(\text { bulk })}\right) d z .
$$

In Eq. (3) $\rho_{\text {seg }}(z)$ is the segment-density profile at a distance $z$ from the attractive surface and $\rho_{\text {seg }}$ (bulk) is the segmentdensity profile in the bulk, i.e., at distance $z$ far from the surface. At infinitely dilute conditions the depletion layer cannot be defined when irreversible adsorption occurs (e.g., when polymer-surface attraction is large). The quantity $\delta$ is positive when a depletion layer is observed and negative when the polymer segments are preferentially adsorbed on the surface. To characterize the properties of polymers that are preferentially adsorbed on the surface, for which $\delta$ assumes physically confusing negative values, we introduce a dimensionless parameter $\Theta$ defined as

$$
\Theta=100\left(0.5-\frac{\delta}{R_{x y z}}\right) .
$$

In Eq. (4) $R_{x y z}$ is the radius of gyration of the polymer in the bulk. The parameter $\Theta$ is positive when polymer segments accumulate close to the surface and negative when the depletion layer is larger than half the radius of gyration. Values of $\Theta$ larger than 50 indicate the absence of a depletion layer. In Table II we report values for $\delta$ and $\Theta$ obtained for the different polymer-surface systems considered in the infinitely dilute continuum model. Results shown in Table II suggest that the polymer architecture has a small influence on the depletion-layer thickness when $\lambda=0.75$ and $\xi=2 k T$ (see Fig. 2 ), and when $\lambda=1.00$ and $\xi=2 k T$. At this $\lambda$ the entropic loss due to adsorption prevails over the enthalpic gain and the surface is effectively repulsive leading to a depletion layer. Our results indicate that $\delta$ is large for linear polymers, decreases for comb polymers with one- or three-segment-long side chains, and then increases as the length of side chain increases. Correspondingly, $\Theta$, negative for linear polymers, increases, reaches a maximum for comb polymers with three-segment-long side chains, and then decreases as the length of the side chains increases.

To assess the effect of adsorption on the statistical properties of the polymers, we compute the three- and the twodimensional (parallel to the surface) radii of gyration of the 
polymers as a function of the distance of the center of mass from the attractive surface. The radii of gyration are obtained as

$$
\begin{aligned}
R_{x y z}^{2}\left(z_{\mathrm{CM}}\right)= & \left\langle\frac { 1 } { N } \sum _ { i = 1 } ^ { N } \left[\left(x_{i}-x_{\mathrm{CM}}\right)^{2}+\left(y_{i}-y_{\mathrm{CM}}\right)^{2}\right.\right. \\
& \left.\left.+\left(z_{i}-z_{\mathrm{CM}}\right)^{2}\right]\right\rangle, \\
R_{x y}^{2}\left(z_{\mathrm{CM}}\right)= & \left\langle\frac{1}{N} \sum_{i=1}^{N}\left[\left(x_{i}-x_{\mathrm{CM}}\right)^{2}+\left(y_{i}-y_{\mathrm{CM}}\right)^{2}\right]\right\rangle .
\end{aligned}
$$

In Eqs. (5) and (6) $N$ is the total number of segments in the polymer; $x_{\mathrm{CM}}, y_{\mathrm{CM}}, z_{\mathrm{CM}}$ are the coordinates of the polymer center of mass; $x_{i}, y_{i}, z_{i}$ are the coordinates of the $i$ th segment; and angular brackets denote ensemble averages. For the sake of brevity, we report the radii of gyration results obtained for the polymers considered in Fig. 2 in the Appendix. Our results (see Appendix, Fig. 9) indicate that as the polymer center of mass approaches the attractive surface, $R_{x y z}^{2}$ [Fig. 9(a)] slightly decreases and $R_{x y}^{2}$ increases [Fig. 9(b)]. The increase in $R_{x y}^{2}$ is a clear indication of the tendency for the adsorbed polymers to spread on the surface. When the center of mass is located at $z<5$ the polymers appear to collapse, but it should be noted that only a few polymers are located at these distances from the surface.

In order to understand if the entropic loss upon adsorption is the limiting factor that effectively repels the polymer segments from the surface, we repeat the simulations discussed above for larger values of $\lambda$. As $\lambda$ increases, the entropic loss upon adsorption should decrease because adsorbed polymers can assume several different conformations while maintaining the same surface-polymer favorable energy. In Fig. 3 we present results for the segment-density profiles for linear [Fig. 3(a)], comb polymers with fivesegment-long side chains [Fig. 3(b)], and comb polymers with eight-segment-long side chains [Fig. 3(c)] when $\xi$ $=2 \mathrm{kT}$ and $\lambda$ equals 1.0 (black solid lines) and 1.5 (black dotted lines). As $\lambda$ increases the density of polymer segments near the surface increases, the depletion layer thickness decreases, and the parameter $\Theta$ increases (see Table II). The effect is particularly evident for the comb polymer with fivesegment-long side chains [Fig. 3(b)]. The results shown in Fig. 3 lead us to conclude that the entropy loss limits the adsorption of polymers at solid surfaces and that adsorption increases when the entropy loss due to adsorption is somehow reduced.

To test the relative importance of enthalpic and entropic driving forces for polymer adsorption, we fix $\lambda=1$ and we simulate the adsorption of comb polymers at solid surfaces varying $\xi$ between 2,3 , and $4 k T$. In addition to the density profile of the polymer segments we also compute the density profile of the centers of mass of the polymers, $\rho_{\text {com }}(z)$, which is defined as
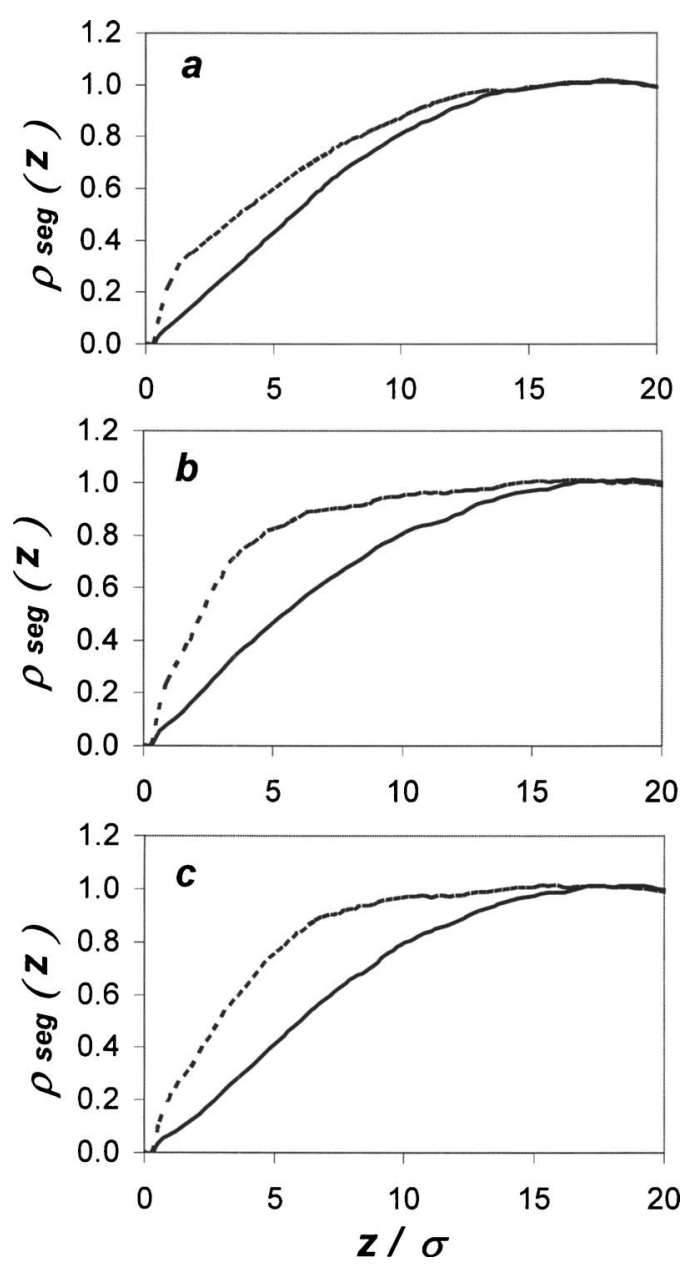

FIG. 3. Segment-density profiles for linear polymers (a), comb polymers with five-segment-long side chains (b), and comb polymers with eightsegment-long side chains (c) near an attractive surface. For all polymers $N_{\text {backbone }}=99$. In all cases $\xi=2 k T ; \lambda=1.0$ (black solid line) or $\lambda=1.5$ (black dotted line). The percentage error in these data is below $10 \%$.

$$
\rho_{\text {com }}(z)=\frac{P(z)}{P(\text { bulk })} .
$$

In Eq. (7) $P(z)$ is the density of the centers of mass of polymers located at a separation $z$ from the surface. From the values of $\rho_{\text {com }}(z)$ we can understand if one polymer is effectively attracted to or repelled from the surface. When $\rho_{\text {com }}(z)>1$ the polymers whose center of mass is located at distance $z$ are effectively attracted to the surface. When $\rho_{\text {com }}(z)<1$ the polymers are effectively repelled.

In Fig. 4 we report results for segment-density profiles [Fig. 4(a)], density profiles of the centers of mass [Fig. 4(b)], and radii of gyration as a function of the distance between the center of mass of the polymer and the surface [Figs. 4(c) and 4(d)] for linear polymers (black solid line), comb polymers with three-segment-long side chains (gray dashed line), and comb polymers with ten-segment-long side chains (black dotted line) when $\xi=4 k T$ and $\lambda=1$. For all polymers $N_{\text {backbone }}=99$. The segment-density profiles shown in Fig. 4(a) indicate that when we consider large attractive polymersegment-surface interactions the depletion layer disappears, leading to large positive values of the parameter $\Theta$ (see Table II for $\xi=4 k T$ and $\lambda=1$ ). This significant accumulation of 

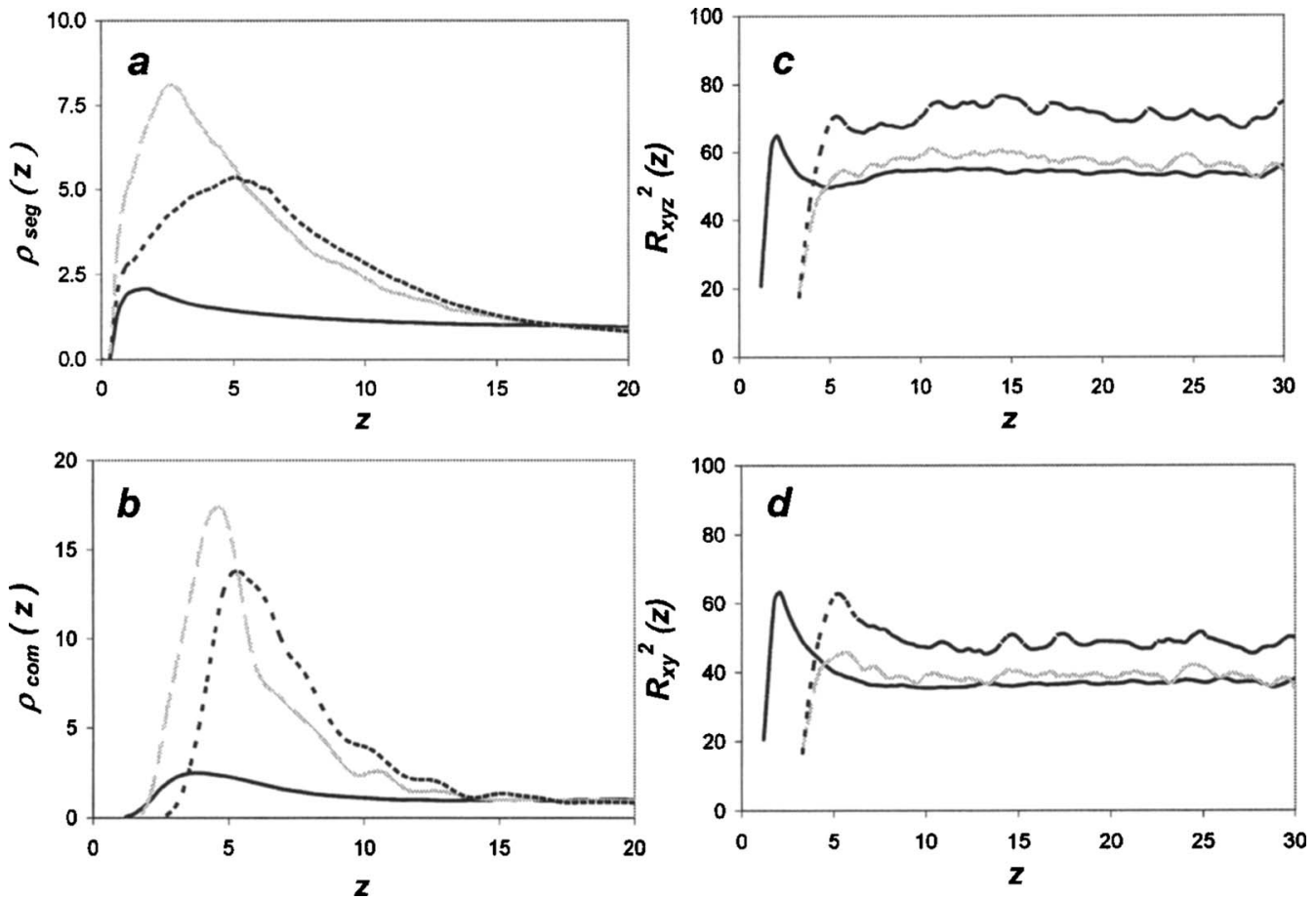

FIG. 4. Segment-density profiles (a), density profiles of centers of mass of polymers (b), three- and two-dimensional radii of gyrations squared [(c) and (d), respectively] for linear polymers (black solid lines), comb polymers with three-segment-long side chains (gray dashed lines), and comb polymers with ten-segment-long side chains (black dotted lines) near an attractive surface when $\lambda=1.0$ and $\xi=4 k T$. For all polymers $N_{\text {backbone }}=99$.

polymer segments is also manifested in an effective surfacepolymer attraction at distances larger than three to four as indicated by the density profiles of the centers of mass of the polymers [Fig. 4(b)]. Results for the radii of gyration [Figs. 4(c) and 4(d)] suggest that the adsorbed polymers readily spread on the surface. Comparing the results obtained upon increasing $\lambda$ while keeping $\xi$ constant (Fig. 3) to the results obtained upon increasing $\xi$ while maintaining $\lambda$ constant (Fig. 4), we note that polymer adsorption increases more significantly when $\xi$ increases than when $\lambda$ increases. By realizing that an increase in $\xi$ corresponds to an increase in the enthalpy of adsorption and that an increase in $\lambda$ corresponds to a decrease in the entropy loss upon adsorption, we conclude that enthalpy is the driving force for adsorption to occur. The entropy loss limits polymer adsorption, but this limitation can be overcome by appropriate increases in the polymer-segment-surface attractive interaction.

On weakly adsorbing surfaces we observe similar results, but less pronounced (for the sake of brevity the results are reported in Appendix, Fig. 10). Our results lead us to conclude that short side chains promote the adsorption of polymers on solid surfaces.

For the set of polymer-surface interaction parameters $\xi$ $=3 \mathrm{kT}$ and $\lambda=1$ we investigate the effect of polymer length on the behavior of the polymers near an attractive surface. We change the number of polymer segments in the polymer backbone, while maintaining the total number of attractive segments per polymer molecule constant. Results for the segment-density profiles near the attractive surface for polymers with $N_{\text {backbone }}=69$ or 129 segments confirm that the presence of short side chains promotes the adhesion of polymers to an attractive surface (results not shown graphically for brevity, but reported in Table II). In addition, we find that the length of the side chains that maximizes polymer adsorption increases as $N_{\text {backbone }}$ increases. Comb polymers with $N_{\text {backbone }}=69$ maximize the parameter $\Theta$ when $N_{\text {side chain }}=3$ while comb polymers with $N_{\text {backbone }}=129$ maximize the parameter $\Theta$ when $N_{\text {side chain }}=5$ (see Table II).

\section{Adsorption mechanism}

To visualize the molecular mechanism of polymer adsorption, we show selected snapshots for adsorbed polymers when $\xi=4 k T$ and $\lambda=1$ in Fig. 5. All the polymers shown in Fig. 5 [linear polymers (left panel), comb polymers with three-segment-long side chains (central panel), and comb polymers with ten-segment-long side chains (right panel)] have $N_{\text {backbone }}=99$. We represent the attractive surface with the black dotted lines, the attractive polymer segments with

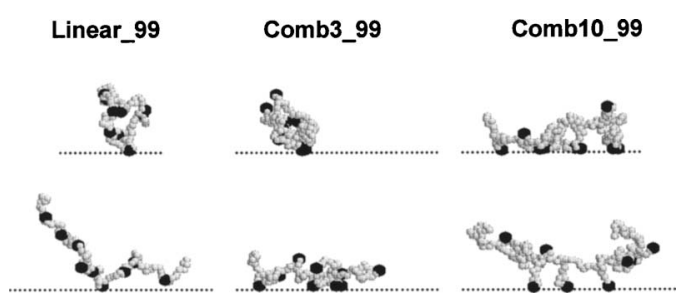

FIG. 5. Representative snapshots for polymers adsorbed on a solid surface when $\lambda=1.0$ and $\xi=4 k T$. Polymers considered have $N_{\text {backbone }}=99$ and are either linear (left panels), comb polymers with three-segment-long side chains (center panels), or comb polymers with ten-segment-long side chains (right panels). The black dotted lines represent the solid surfaces. The black and gray spheres represent attractive and nonattractive polymer segments, respectively. 

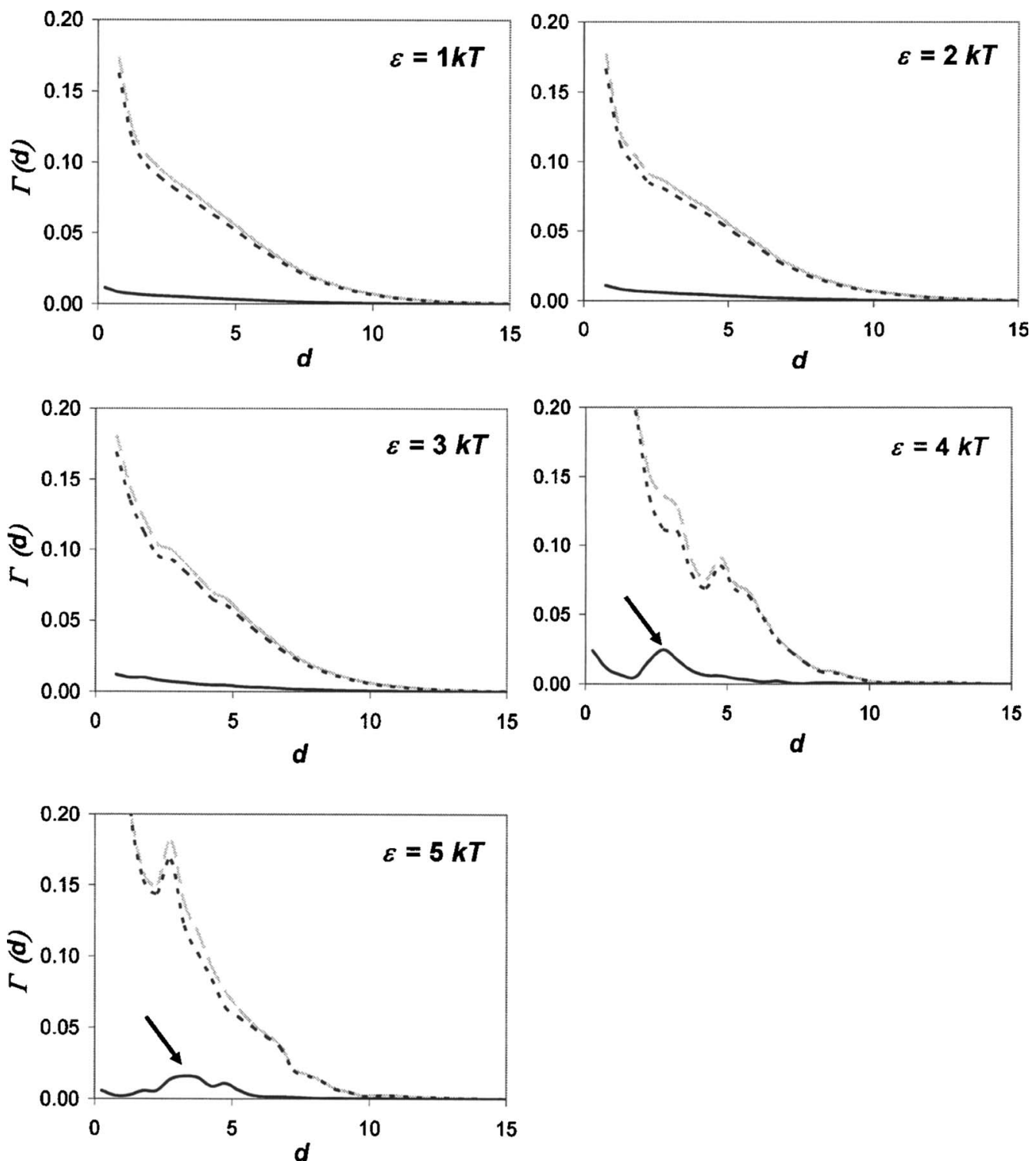

FIG. 6. Segment density, $\Gamma$, of all segments (gray dashed lines), attractive segments (black solid lines), and nonattractive segments (black dotted lines) as a function of the distance from the center of mass, $d$, for comb polymers with $N_{\text {backbone }}=99$ and three-segment-long side chains for different values of the polymer-segment-segment interaction parameter $\varepsilon$. The arrows indicate the density maxima for the attractive segments discussed in the text.

black spheres, and the nonattractive polymer segments with gray spheres. The snapshots indicate that upon adsorption the comb polymers project their side chains towards the surface and maintain their backbones within the bulk solution to maximize both the entropy and the enthalpy of adsorption. For linear polymers the adsorbed conformations become entropically disadvantageous. For comb polymers with long side chains, because of the limited number of attractive segments, enthalpically favorable polymer-segment-surface contacts can be established when the majority of the polymer segments on the backbone remain far from the surface to maximize the system entropy. Because of these two limiting behaviors, there is a competition between the tendency of the polymers to remain in the bulk solution and to adsorb onto the surface. Our results indicate that optimum conditions for adsorption are obtained when the polymers have short side chains (three segments when $N_{\text {backbone }}=99$ ).

\section{Bulk versus adsorbed configurations}

Our results at infinitely dilute concentrations suggest that adsorption of a polymer on a surface is promoted when it has short side chains. To better appreciate the change in conformation that these polymers experience when they are adsorbed on a surface from the bulk, we further study the bulk properties of a comb polymer with $N_{\text {backbone }}=99$ and $N_{\text {side chain }}=3$ at varying polymer-segment-segment interaction. The surface is not considered in these calculations. The strengths of the square-well potential parameter $\varepsilon$ in Eq. (1) are equal to $1,2,3,4$, and $5 k T$. Large values of $\varepsilon$ indicate low temperatures, at which polymer-segment-segment attractive interactions become significant.

In Fig. 6 we report results for the density of polymer segments, $\Gamma$, as a function of the distance from the polymer center of mass at infinitely dilute concentrations showing the total segment density (gray dashed lines), the density of the attractive segments (black solid lines), and the density of the 
nonattractive segments (black dotted lines). We report the radius of gyration for the bulk polymers considered in Fig. 6 in the Appendix, Table III. Results shown in Table III indicate that as $\varepsilon$ increases, the radius of gyration of the polymers decreases. We note that when $\varepsilon=1 k T$ the segmentdensity profiles decay smoothly as the distance from the center of mass increases, but when $\varepsilon=4$ or $5 k T$ they decay irregularly and abruptly, suggesting that at these conditions the polymers collapse into well-defined globular conformations. In particular, when $\varepsilon=5 k T$ the polymer radius of gyration squared collapses to one-half of the value observed when $\varepsilon=1 k T$ (see Table III). This result is quite surprising when we notice that the number of attractive segments (black solid lines in Fig. 6) is less than $10 \%$ of the total segments in the polymer (gray dashed lines in Fig. 6). However, contrary to our expectations, the attractive segments(black solid lines) do not agglomerate near the center of mass of the polymer when $\varepsilon=4$ or $5 k T$ but rather accumulate at some distance from it. In fact, at $\varepsilon=4 k T$ and at $\varepsilon=5 k T$ the black solid line has a maximum at a distance equal to three to four $\sigma$ from the polymer center of mass (see arrows in Fig. 6), and not near the center of mass as observed, for example, for $\varepsilon$ $=1 k T$. Further studies are necessary to determine if the polymers considered here have first-order disorder-to-order transitions as a function of temperature (or $\varepsilon)^{30,31}$

The results shown in Fig. 6, as well as simulation snapshots (not shown for brevity), indicate that the majority of the attractive segments are buried within the globular bulk conformation of the polymers at each value of $\varepsilon$ considered, even though they are not accumulated at the center of mass. For example, when $\varepsilon=4 k T$ and $\varepsilon=5 k T$ the total segment density (gray dashed lines in Fig. 6) is nonzero at distances larger than those at which the density of the attractive segments (black solid lines in Fig. 6) equals zero. This conformation is similar to that of aqueous globular proteins in which the hydrophobic residues are preferentially buried within the hydrophobic interior of the protein. ${ }^{32}$ This suggests that our model can be used as a first approximation to study the adsorption of complex macromolecules on attractive surfaces. It should, however, be noted that our simple model cannot capture the formation of secondary protein structures that can only be described by complicated models such as those discussed by Smith and Hall, ${ }^{33}$ and Leonhard et $a l .{ }^{34}$

\section{B. Finite-concentration lattice model 1. Single chain}

In order to facilitate the comparison between results obtained using the infinitely dilute continuum algorithm and the finite-concentration lattice model, we perform lattice model simulations of a system containing only one polymer molecule $\left(N_{\text {polymers }}=1\right)$. The backbone of the polymer is $99 \mathrm{seg}$ ment long. The packing fraction of the polymer segments for the systems considered ranges from 0.0022 for linear polymers to 0.0042 for comb polymers with ten-segment-long side chains. For the sake of brevity, the segment-density profiles for the single polymer chain, together with calculation details, are shown in the Appendix, Fig. 11. While the results differ from those obtained with the infinitely dilute continuum model (see Appendix for details), we observe that the presence of short side chains promotes polymer adsorption on the surface. This observation ensures that the choice of parameters in the finite-concentration lattice model is such that it can qualitatively reproduce the systems simulated in the infinitely dilute continuum model.

Analogous to the parameter $\Theta$ discussed in the infinitely dilute continuum model, we calculate the polymer-segmentsurface excess, $Z^{*}$, defined as ${ }^{35}$

$$
Z^{*}=\int_{0}^{\infty}\left(\frac{\rho(z)}{\rho(\text { bulk })}-1\right) d z
$$

In Eq. (8) $\rho(z)$ is the segment density at a distance $z$ from the attractive surface and $\rho$ (bulk) is the segment density in the bulk far from the surface. The limits of the integral in Eq. (8) run from $z=0$ to $z \rightarrow \infty$ because $\rho(z) \rightarrow \rho$ (bulk) as $z \rightarrow \infty$. Because we have a finite simulation box we cannot calculate the surface excess as $z \rightarrow \infty$. Instead in this finiteconcentration lattice model we conduct the integration from $z=0$ (location of the attractive surface) to $z=25$ (far from both the attractive surface at $z=0$ and the hard surface at $z$ $=50$ ) and therefore $\rho$ (bulk) is set equal to $\rho(25)$. Positive values of the parameter $Z^{*}$ indicate an accumulation of polymer segments on the surface and therefore the parameter $Z^{*}$ is analogous to the parameter $\Theta$ defined in the infinitely dilute continuum model [see Eq. (4)]. Negative values of the parameter $Z^{*}$ are interpreted as a depletion layer near the surface since algebraically the parameter $Z^{*}$ is the negative of the width of the depletion layer $\delta$ [see Eq. (3)]. The inset in Fig. 11(a) shows the average values of $Z^{*}$ obtained for the different architectures of the polymers in the finiteconcentration lattice model when a single polymer is dissolved within the simulation box. Our results indicate that $Z^{*}$ is negative for linear polymers (indicating the presence of a depletion layer), increases and reaches a maximum for comb polymers with one-segment-long side chains, and then decreases as the length of the side chains increases. When the side chains are five segments or longer, the density profiles, within statistical uncertainties, do not change significantly and are similar to those obtained for linear polymers. These results are in qualitative agreement with the results obtained in the infinitely dilute continuum model where, as the length of the side chains increases, the parameter $\Theta$ increases, reaches a maximum, and then decreases [inset in Fig. 10(a)]. The difference between the two models is that in the finiteconcentration lattice model the comb polymer with onesegment-long side chains has the highest $Z^{*}$ while in the infinitely dilute case the comb polymer with three-segmentlong side chains has the highest $Z^{*}$.

\section{Multiple chains}

Next, we increase the concentration of the polymers within the simulated systems. We consider systems in which 20 polymers are dissolved within the simulation box. The corresponding packing fraction of the polymer segments 

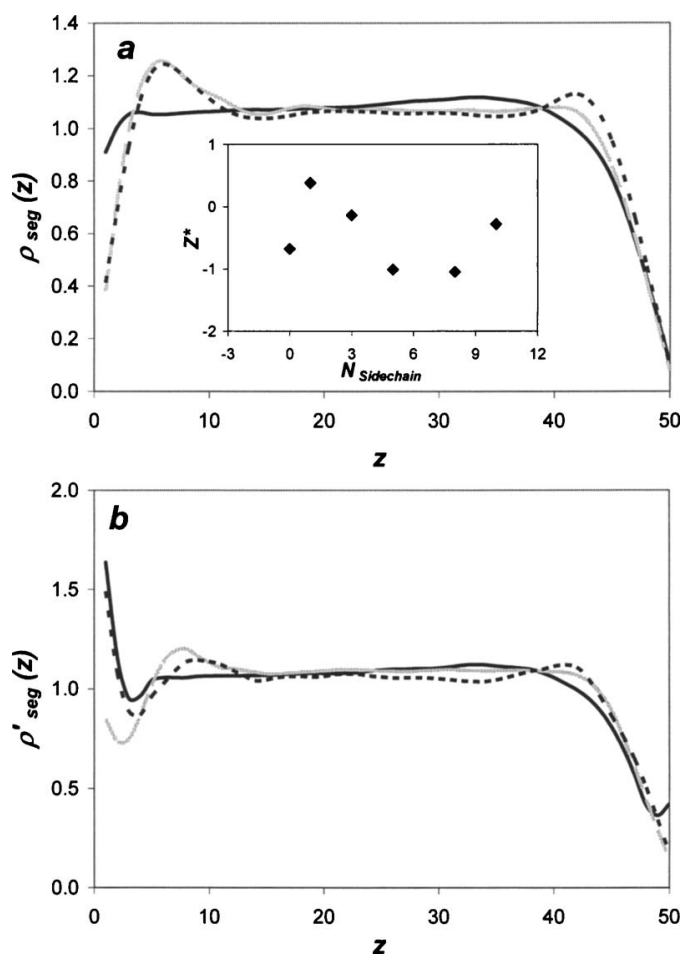

FIG. 7. Total segment density, $\rho_{\text {seg }}(z)$, (a) and segment density of attractive segments, $\rho_{\mathrm{seg}}^{\prime}(z)$, (b) for a system containing 20 polymers as a function of distance from the surface, $z$, for linear polymers (black solid lines), comb polymers with three-segment-long side chain [gray dashed lines, only (a)], comb polymers with five-segment-long side chain [gray dashed lines, only (b)], and comb polymers with ten-segment-long side chain (black dotted lines) using the finite-concentration lattice model.

ranges from 0.044 for linear polymers to 0.084 for comb polymers with ten-segment-long side chains. While the polymer number density (ratio of number of polymers to the volume of the simulation box) remains constant as the length of the side chains increases, the packing fraction of the polymer segments increases, and the systems with comb polymers with ten-segment-long side chains are almost twice as packed as those with linear polymers.

Results obtained for a system containing 20 polymers $\left(N_{\text {polymers }}=20\right)$ are displayed in Fig. 7. In Fig. 7(a) we show the total segment-density profiles for linear (black solid lines), comb polymers with three-segment-long side chains (gray solid lines), and comb polymers with ten-segment-long side chains (black dotted lines). The inset in Fig. 7(a) shows the parameter $Z^{*}$ as a function of the length of the side chains in the polymers. Our results indicate that $Z^{*}$ is negative for linear polymers, increases for comb polymers with onesegment-long side chains and three-segment-long side chains, decreases for comb polymers with five-segment-long side chains and eight-segment-long side chains, and increases for ten-segment-long side chains. This observation is qualitatively similar to the results obtained for a single polymer with the finite-concentration lattice model [Fig. 11(a)] where the comb polymer with one-segment-long side chain has the highest value of $Z^{*}$. The notable difference between single chain and multiple chains in the finite-concentration lattice model is that in the multiple-chain case the comb polymer with ten-segment-long side chains has a higher value of $Z^{*}$ than the comb polymers with five-segment- and eight-segment-long side chains. This could be due to both the change in conformations of the chains as the concentration in the simulation box increases and the increased packing fraction of polymer segments. This will be discussed later in this section.

In Fig. 7(b) we report the density profile of attractive segments for linear polymers (black solid line), comb polymers with five-segment-long side chains (gray dashed line), and comb polymers with ten-segment-long side chains (black dotted lines). The density profile of the attractive segments at a distance $z$ from the surface is obtained as the ratio of the average density of attractive polymer segments found at that distance and the average density of attractive polymer segments in the box (equal to the number of attractive segments in the simulation box divided by the box volume). Our results indicate that the number of attractive segments in contact with the surface is the highest for the linear polymer, decreases as the length of the side chain increases, reaches a minimum for the five-segment-long side chain, and increases for comb polymers with ten-segment-long side chains. These results are in conflict with those obtained for a single polymer using the finite-concentration lattice model [Fig. 11(b)] where the number of attractive segments near the surface is highest for comb polymers with three-segment-long side chains and lowest for comb polymers with ten-segment-long side chains. This difference can be attributed to conformational changes of the polymers near the attractive surface upon increasing the polymer concentration.

To better understand the conformations of polymers adsorbed on a surface from a finite-concentration solution we show simulation snapshots (see Fig. 8) of linear polymers (top panel), comb polymers with one-segment-long side chains (middle panel), and comb polymers with ten-segmentlong side chains (bottom panel). For clarity the snapshots show only a few adsorbed chains in each system. The attractive segments in the polymers are represented with large black spheres. The nonattractive segments are represented with spheres of different shades of gray so that the chains can be distinguished from each other. When linear polymers adsorb on a surface (top panel), they interpenetrate each other. In addition, we can see in the snapshots that each individual adsorbed chain spreads on the surface forming "trains" and "loops." Similar conformations are also observed at infinitely dilute concentrations in the continuum model (see Fig. 5, left panels). However, visual inspection of the snapshot shown in Fig. 8 (top) indicates that linear polymers establish a larger number of contacts with the surface in the finite-concentration (lattice model) than at infinitely dilute concentrations. This qualitative observation is supported by results obtained for the density of attractive segments shown in Fig. 7(b).

Comb polymers with one-segment-long side chains (middle panel) from individual globules and adsorb on the surface separately with no evidence of entanglement. Some of the comb polymers with ten-segment-long side chains (bottom panel) stay in individual coils and others interpenetrate during adsorption. As mentioned earlier, the packing fraction of the polymer segments increases as the length of 


\section{Linear}
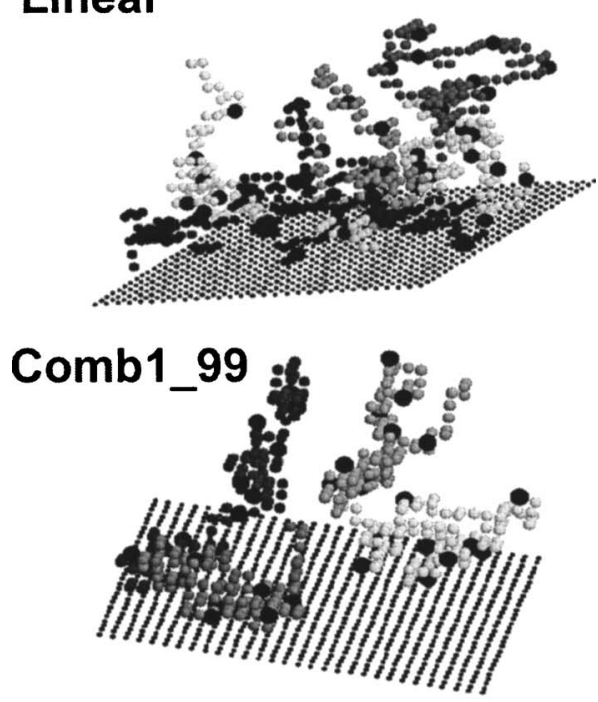

Comb10_99

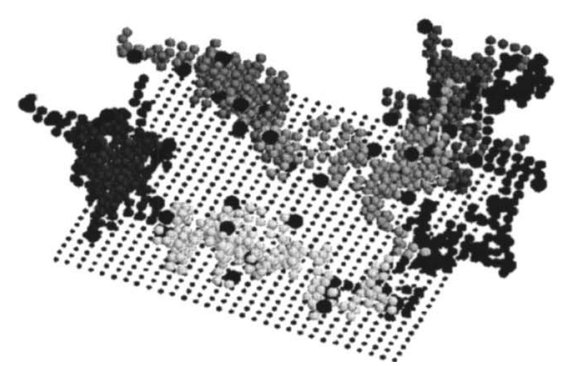

FIG. 8. Representative snapshots for linear polymers (top), comb polymers with one-segment-long side chains (middle), or comb polymers with tensegment-long side chains (bottom) adsorbed on a solid surface using the finite-concentration lattice model. The attractive polymer segments are represented with black spheres. The nonattractive segments on the different chains are represented with different shades of gray.

the side chains increases, and this could explain why comb polymers with ten-segment-long side chains in some cases interpenetrate while comb polymers with one-segment-long side chains do not. In addition, we expect that polymerpolymer interactions play a significant role in determining the conformation of adsorbed polymers at finite concentration. In the literature it has been observed that at dilute bulk concentrations linear polymers interpenetrate, whereas branched polymers repel each other. ${ }^{36}$ Therefore it is expected that linear polymers will adsorb and interpenetrate each other while comb polymers will adsorb as single globules without interpenetrations.

With the aid of the simulation snapshots we can rationalize the differences observed in the results at low concentration (one polymer in the simulation box) and moderate concentrations ( 20 polymers in the simulation box). At low concentration polymers adsorb on an attractive surface for enthalpic reasons. The favorable polymer-surface interactions cause linear polymers and comb polymers with short side chains to adsorb and spread on the surface (see Fig. 5). When the polymer concentration increases, the enthalpic gain due to polymer-segment-surface interactions can also be achieved by favorable segment-segment associations between different polymer chains near the surface. In the case of linear polymers the association between a few polymer chains drives some of the adsorbing linear polymers to entangle and interpenetrate (see Fig. 8, top panel). The linear polymers that do not establish favorable segment-surface interactions or favorable association with other polymers remain as globules in the bulk. This explains why the linear polymers have a lower value of $Z^{*}$ but a higher number of attractive segments adsorbed at the surface at moderate concentration as compared to low concentration.

At moderate concentrations comb polymers with short side chains associate, preferentially in the bulk. Those polymers that do not associate in the bulk adsorb on the surface as individual globules (see Fig. 8, middle panel). This explains why comb polymers with short side chains (one segment long and three-segment long) have lower number of attractive segments on the surface at moderate concentration as compared to low concentration.

Comb polymers with long side chains (10 segment long) adsorb on a surface readily at infinitely dilute concentrations as they do not suffer a large loss in entropy (see Fig. 5). However, our results at dilute concentrations in the lattice model (Fig. 11) show that these polymers do not adsorb on the attractive surface for the set of interaction parameters chosen here. At moderate concentration in the lattice model, as the packing fraction increases, even comb polymers with ten-segment-long side chains adsorb (see Fig. 7). Interestingly, adsorbed conformations of the comb polymers with ten-segment-long side chains are similar to the conformations far from the surface. Many of the polymers adopt globular configurations in which some attractive segments are in contact with the attracting surface and others are in contact with other polymer chains (see Fig. 8 bottom panel). The visual observation of the snapshots shows that the conformation of the polymers does not change upon adsorption. Therefore we conclude that for comb polymers with tensegment-long side chains the adsorption is predominantly driven by the large packing fraction in the simulation box. This system is indeed the most crowded among those studied here, and it is likely that the confined polymers try to occupy the largest possible volume in an attempt to maximize the entropy of the system.

\section{SUMMARY AND CONCLUSIONS}

We have presented a combination of molecularsimulation studies of the adsorption of linear and comb polymers on weakly attractive surfaces. The length of the backbone in the comb polymers is equal to the length of the linear polymers. The molecular weight of the polymers is increased by increasing the length of the side chains in the comb polymers. The majority of the segments comprising a polymer molecule interact with each other and with the surface via hard-sphere potentials. Selected polymer segments, located at the tips of the side chains in comb polymers or equally spaced along linear polymers, interact with each other and with the surface via attractive square-well potentials. This 
architecture is chosen to crudely represent proteins, in which associating interactions between amino acids are primarily responsible for the secondary and tertiary structures. In our simplistic representation, proteins are represented as comb polymers with short side chains. We are interested in understanding if this simple representation of protein architecture determines the thermodynamic properties of proteins (e.g., adsorption on hydrophobic surfaces). We investigate the effect of the length of the side chains on the properties of the adsorbed polymers.

At infinitely dilute concentrations short side chains promote adsorption because they allow the polymer molecules to maximize the enthalpic gain due to the adsorption of attracted segments, and minimize the entropic penalty faced during adsorption. We note that, among the polymers considered here, comb polymers with short side chains resemble aqueous proteins more closely than the other polymer architectures do. Our results suggest that the architecture of proteins is responsible for the tendency of proteins to adsorb and degenerate on top of hydrophobic surfaces. Our results seem to suggest that this architecture is optimal for the synthesis of polymers that are required for adsorption on solid surfaces and formation of brushes. Interestingly, this polymer architecture (comb polymers with short side chains) has been used by Auguste et al. ${ }^{5}$ to coat liposomes with polymer brushes. We find that long side chains do not promote the spreading of polymers on the attractive surface because the free energy of adsorption is minimized when the attractive segments are in contact with the surface (maximizing the enthalpy gain due to adsorption) and the nonattractive segments are far from the surface (maximizing the entropy of the system). Snapshots of the molecular configurations taken during the simulation show that comb polymers undergo a significant conformational change upon adsorption. When comb polymers are dissolved in bulk solutions at low temperatures, the attractive segments preferentially form clusters near the center of mass of the polymer, but when the comb polymers are adsorbed onto an attractive surface the attracted segments are preferentially in contact with the attractive surface while the backbone remains in solution. This conformational change resembles that observed when aqueous proteins are adsorbed onto hydrophobic surfaces. Further, by varying the width and depth of polymer-segment-surface square-well potentials we demonstrate that the driving force for adsorption to occur is the enthalpic gain, while the entropic loss constitutes an obstacle to adsorption that can be overcome by either favorable polymer architectures or by increased surface-polymer attraction. The results obtained at infinitely dilute concentrations are in agreement with the results obtained at low concentration using a lattice model where the comb polymer with short side chains promoted the adsorption of polymers onto an attractive surface. However, in the lattice model at low concentration the comb polymer with ten-segment-long side chains did not adsorb on the attractive surface. For this specific polymer architecture the set of interaction parameters chosen in the lattice model is probably such that the enthalpic gain due to adsorption does not compensate for the entropic loss, and consequently the polymer does not adsorb.
At moderate concentration the enthalpic gain due to polymer-segment-surface interactions is achieved by favorable segment-segment associations between different polymer chains near the surface. Linear polymers adsorb on an attractive surface and they form "trains" in which several attractive segments are in contact with the surface. Linear polymers are flexible and they easily interpenetrate each other. Our simulation snapshots suggest that by interpenetrating each other the linear polymers minimize the free energy of the system by establishing contacts between attractive segments belonging to different polymers (thus minimizing the system enthalpy) and by allowing several possible conformations (thus maximizing the system entropy). In contrast, comb polymers repel each other, and rarely interpenetrate. At moderate concentration comb polymers with short side chains form globules in which the attractive segments are often located at the exterior. This configuration promotes the formation of contacts between attractive segments belonging to different polymers, or between attractive segments and the attractive surface, in an attempt to lower the global free energy. As a consequence, we observe that adsorbed polymers have a few attractive segments in contact with the surface and a few others projected towards the center of the simulation box. This behavior is in contrast with our results at infinitely dilute concentrations (continuum model) or at low concentrations (lattice model) where the majority of the attractive segments is found in contact with the attractive surface and the polymer backbone is projected towards the center of the simulation box. We attribute the difference to the absence of the possibility of establishing contacts between attractive segments belonging to different polymers at dilute or infinitely dilute concentrations. Comb polymers with ten-segment-long side chains at moderate concentrations adsorb on the attractive surface, even though they do not adsorb at low concentration (lattice model). The conformation of comb polymers with long side chains does not change appreciably when the concentration increases. Furthermore the conformations of adsorbed chains are not different from that of the chains in the bulk. In a typical conformation, the attractive segments are preferentially located on the exterior of a globule of polymer segments. Our results indicate that these globules adsorb on the attractive surface at large packing fraction. Since the conformations of the polymers do not change appreciably upon adsorption we speculate that at the conditions considered here adsorption occurs only because of steric effects.

\section{ACKNOWLEDGMENTS}

Financial support for this work was partially provided by the US National Science Foundation under Grant No. DMR0103399 and the US Department of Energy under Grant No. DE-FG05-91ER14181. The infinitely dilute continuum simulations were conducted at the Center for Advanced Computing at the University of Michigan through a NPACI grant. 

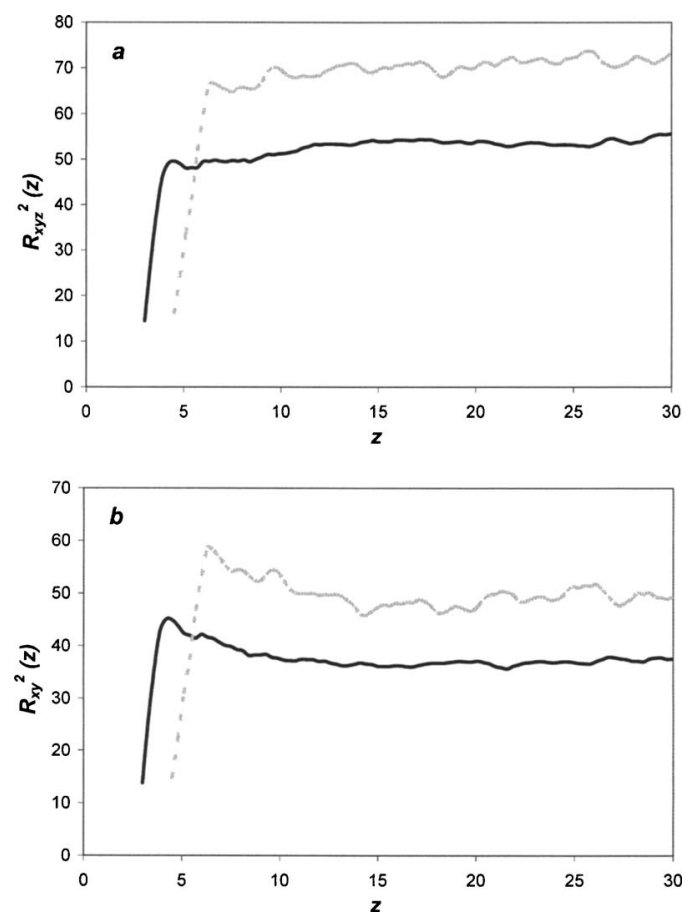

FIG. 9. Three- $\left[R_{x y z}^{2},(\mathrm{a})\right]$ and two-dimensional $\left[R_{x y}^{2},(\mathrm{~b})\right]$ radii of gyration squared as a function of the center-of-mass distance from the attractive surface for linear polymers (black solid line) and comb polymers with tensegment-long side chains (gray dashed line) when $\xi=2 k T$ and $\lambda=0.75$. For both polymers $N_{\text {backbone }}=99$. The percentage error in these data is below $5 \%$.

\section{APPENDIX}

\section{Infinitely dilute continuum model: Statistical conformations of polymers near surfaces}

Results shown in Fig. 9.

\section{Infinitely dilute continuum model: Polymers adsorbed on weakly attractive surfaces}

To investigate the effect of polymer architecture on adsorption when polymers are weakly adsorbed on a surface, we simulate the adsorption of comb polymers on solid surfaces when $\xi=3 k T$ and $\lambda=1$. We report the segment-density profile [Fig. 10(a)] and the density profiles of the centers of mass of the polymers [Fig. 10(b)] for various polymer architectures. At these conditions linear polymers (black solid lines) are effectively repelled from the surface and a wide depletion layer is observed (see Table II for $\xi=3 k T$ and $\lambda$ $=1$ ). As the length of the side chains increases the parameter $\Theta$ increases, becomes positive, reaches a maximum, and then decreases [see inset in Fig. 10(a)]. The maximum in $\Theta$ is obtained when the side chains are three segment long. In agreement with results discussed earlier (see Fig. 2), the results shown in Fig. 10 lead us to conclude that short side chains promote the adsorption of polymers on solid surfaces.

\section{Infinitely dilute continuum model: Polymer radius of gyration in bulk}

Results shown in Table III.
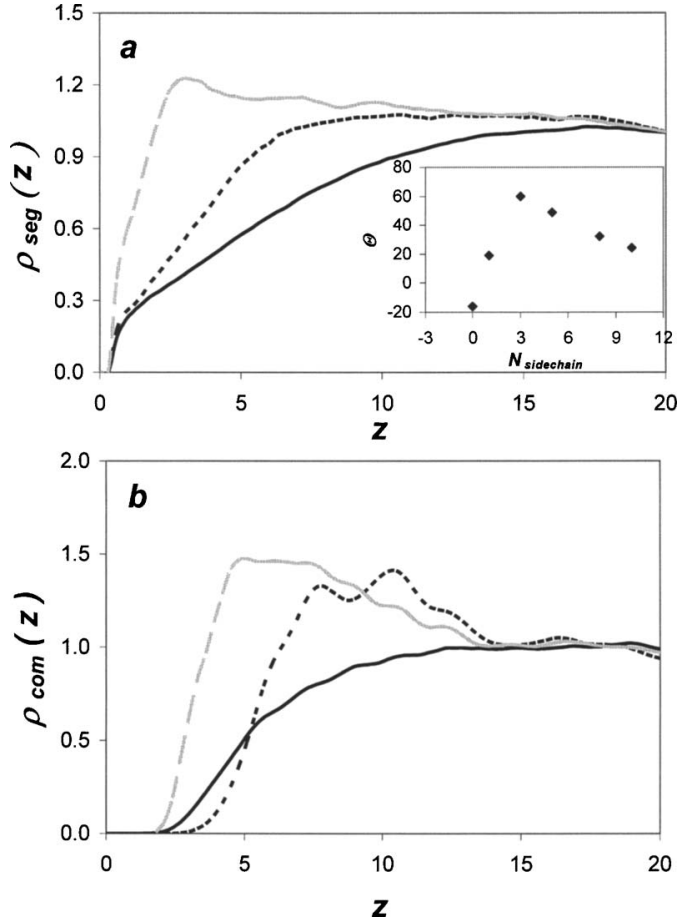

FIG. 10. Segment-density profiles (a) and density profiles of centers of mass of polymers (b) for linear polymers (black solid lines), comb polymers with three-segment-long side chains (gray dashed lines), and comb polymers with ten-segment-long side chains (black dotted lines) near an attractive surface when $\lambda=1.0$ and $\xi=3 k T$. For all polymers $N_{\text {backbone }}=99$. The inset in Fig. 6 shows the parameter $\Theta$ (see Table II) as a function of the number of segments in the side chains. Linear polymers are represented as polymers with side chains of length zero segment.

\section{Finite-concentration lattice model: Single chain}

The total segment-density profile at a distance $z$ from the surface [Fig. 11(a)] is obtained as the ratio of the average density of polymer segments found at distance $z$ and the average density of polymer segments in the box [equal to the ratio of the total number of segments in the simulation box $\left(N_{\text {polymers }} \times N_{\text {total }}\right)$ and the volume of the simulation box]. Only the results for linear polymer (black solid lines), comb polymer with three-segment-long side chains (gray dashed lines), and comb polymer with ten-segment-long side chains (black dotted lines) are shown for clarity. The total segment density of the linear polymer (black solid line) and the comb polymer with ten-segment-long side chains (black dotted line) is low adjacent to the attractive surface $(z=0)$ and then gradually increases as $z$ approaches the center of the simulation box, which is in qualitative agreement with results obtained with the continuum model (see Fig. 10). However, in

TABLE III. Radius of gyration, $\left\langle\boldsymbol{R}_{x y z}{ }^{2}\right\rangle$. for comb polymers with $N_{\text {backbone }}$ $=99$ and 3 -segment-long side chains as a function of the polymer-segmentsegment interaction potential parameter $\varepsilon$ [see Eq. (1)].

\begin{tabular}{cc}
\hline \hline$\varepsilon / k T$ & $\left\langle\boldsymbol{R}_{x y z}{ }^{2}\right\rangle$ \\
\hline 1 & $61.7 \pm 0.2$ \\
2 & $62.2 \pm 0.8$ \\
3 & $56.4 \pm 0.8$ \\
4 & $40.6 \pm 3.2$ \\
5 & $31.6 \pm 1.9$ \\
\hline
\end{tabular}



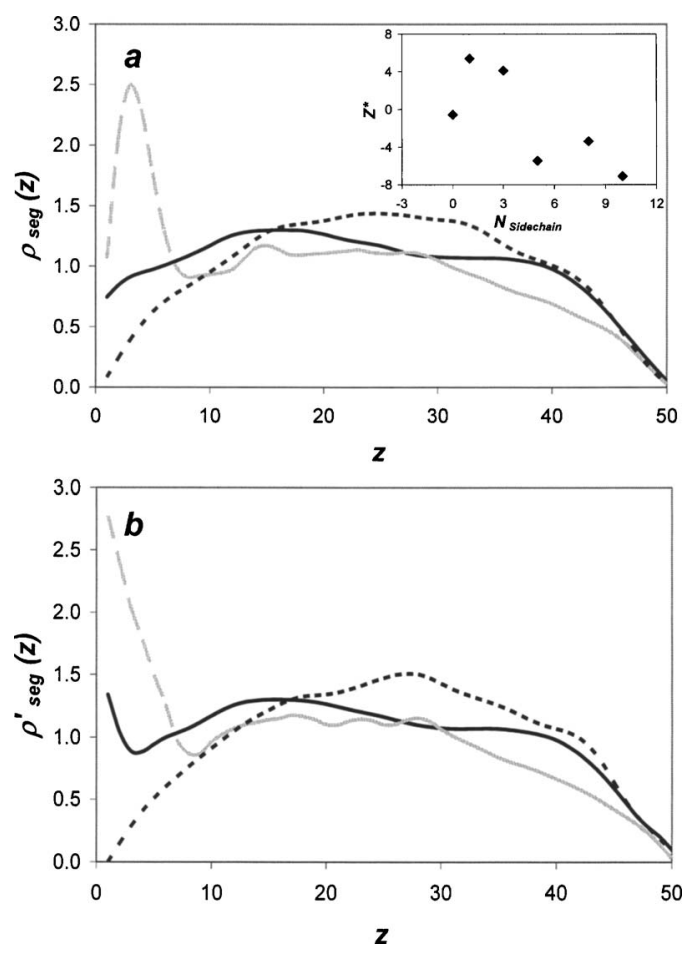

FIG. 11. Total segment density $\rho_{\text {seg }}(z)$ (a) and segment density of attractive segments $\rho_{\mathrm{seg}}^{\prime}(z)$ (b) as a function of distance from the surface, $z$, for single linear polymer (black solid lines), comb polymer with three-segment-long side chain (gray dashed lines), and comb polymer with ten-segment-long side chain (black dotted lines) using the finite-concentration lattice model.

the lattice model [Fig. 11(a)] the total segment density near the surface is larger for the linear polymer (black solid line) than for the comb polymer with ten-segment-long side chains (black dotted line). This result disagrees with the results obtained in the continuum model where the total segment density near the attractive surface is the least for the linear polymer. This difference can be explained with the density profile for the attractive segments [Fig. 11(b)]. While several attractive segments of the linear polymer adsorb on the surface, the attractive segments of the comb polymer with ten-segment-long side chains rarely adsorb. We speculate that in the case of the linear polymer the entropy loss due to adsorption is lower in the lattice model than in the continuum model while in the case of the comb polymer with ten-segment-long side chains the set of interaction parameters chosen for our simulations favors the formation of globules that do not adsorb on the attractive surface.

The total segment density of the comb polymer with three-segment-long side chains near the surface [Fig. 11(a)] is higher than those of the linear polymer and the comb polymer with ten-segment-long side chains. This qualitatively agrees with the results in Fig. 6(a) where the comb polymer with three-segment-long side chains has the highest total segment density near the surface. The results in Fig. 11(a) indicate that the density profile is close to 1 at $z=1$, adjacent to the attractive surface, increases to 2.5 at $z=3-4$, and then decreases to 1 as $z$ approaches the center of the simulation box. Similar features are observed in the continuum model [see Fig. 10(a)], however, the total segment density adjacent to the surface is lower and the peak located at $z=3-5$ is weaker in the continuum model than in the lattice model. These differences can be explained with the density profile for the attractive segments shown in Fig. 11(b). The attractive segments of the comb polymer with three-segment-long side chains accumulate on the surface. This could be a consequence of the shorter range of the segment-surface attractive interaction in the lattice model as compared to that used in the continuum model.

In Fig. 11(b) we plot the density profile of the attractive segments for a single polymer within the simulation box. Our results indicate that when a linear polymer is dissolved within the simulation box [black solid line in Fig. 11(b)] the attractive segments are distributed fairly uniformly across the simulation box. The attractive segment density is largest adjacent to the attractive surface (indicating polymer adsorption), decreases to a minimum at short separations from the attractive surface, and then reaches a plateau (within computational uncertainties) in the center of the simulation box. A similar behavior is observed for a comb polymer with threesegment-long side chains [gray dashed line in Fig. 11(b)]. The minimum in the density profile of the attractive segments observed at $z=4-5$ for the linear polymer or at $z$ $=8-9$ for the comb polymer with three-segment-long side chains indicates that the attractive segments prefer being adsorbed on the surface or being around the center of the simulation box, but they do not like to be at intermediate separations. This is probably a consequence of the fact that the global free energy is minimized when the attractive segments are adsorbed at the surface or are present in the bulk where the number of possible polymer configurations is maximized.

A different behavior is observed for a comb polymer with long side chains $\left[N_{\text {side chain }}=10\right.$ in Fig. 11(b), black dotted line]. In that case the attractive segment density is low close to the attractive surface $(z=0)$ and to the hard surface $(z=50)$ and reaches a maximum in the center of the simulation box. These features are in partial agreement with the configurations of linear and comb polymers adsorbed on attractive solid surfaces in the infinitely dilute continuum model (Fig. 5). The models agree in that they both predict that linear polymer and comb polymers with short side chains readily adsorb and spread on attractive surfaces leading to high densities of attractive segments close to the attractive surface. The models disagree in the following way. The infinitely dilute continuum model predicts that comb polymers with long side chains project their attractive segments towards the attractive surface but withhold their backbone from it (Fig. 5, right panels). The finite-concentration lattice model predicts that the attractive segments of the comb polymers with ten-segment-long side chains are not adsorbed on the attractive surface [see also values for $Z^{*}$ in the inset of Fig. 11(a)]. This could be because they maintain globular conformations, even when close to the attractive surface.

\footnotetext{
${ }^{1}$ P. C. Hiemenz and R. Rajagopalan, Principles of Colloid and Surface Chemistry, 3rd ed. (Marcel Dekker, New York, 1987).

${ }^{2}$ J.-F. Le Meins and J.-F. Tassin, Colloid Polym. Sci. 281, 283 (2003).

${ }^{3}$ U. Reichert, T. Linden, G. Belfort, M.-R. Kula, and J. Thömmes, J. Membr. Sci. 199, 161 (2003).

${ }^{4}$ J. F. Hester and A. M. Mayes, J. Membr. Sci. 202, 119 (2002).
} 
${ }^{5}$ D. T. Auguste, R. K. Prud'homme, P. L. Ahl, P. Meers, and J. Kohn, Biochim. Biophys. Acta 1616, 184 (2003).

${ }^{6}$ I. Norde, in Surface and Interfacial Aspects of Biomedical Applications, edited by J. D. Andrade (Plenum, New York, 1995).

${ }^{7}$ M. A. C. Stuart, G. J. Fleer, J. Lyklema, W. Norde, and J. Scheutjens, Adv. Colloid Interface Sci. 34, 477 (1991).

${ }^{8}$ A. A. Mungikar and D. Forciniti, ChemPhysChem 3, 993 (2002).

${ }^{9}$ A. K. Chakraborty and M. Tirrell, MRS Bull. 21, 28 (1996).

${ }^{10}$ L. G. Cascão Pereira, A. Hickel, C. J. Radke, and H. W. Blanch, Biotechnol. Bioeng. 78, 595 (2002).

${ }^{11}$ F. Fang and I. Szleifer, Biophys. J. 80, 2568 (2001).

${ }^{12}$ F. Fang and I. Szleifer, J. Chem. Phys. 119, 1053 (2003).

${ }^{13}$ M. A. Carignano and I. Szleifer, Mol. Phys. 100, 2993 (2002).

${ }^{14}$ S. S. Sheiko and M. Möller, Chem. Rev. (Washington, D.C.) 101, 4099 (2001)

${ }^{15}$ P. Bauerle, T. Fisher, B. Bidlingmeier, A. Stabel, and J. P. Rabe, Angew. Chem., Int. Ed. Engl. 34, 303 (1995).

${ }^{16}$ S. A. Prokhorova, S. S. Sheiko, A. Mourran et al., Langmuir 16, 6862 (2000)

${ }^{17}$ S. S. Sheiko, S. A. Prokhorova, K. L. Beers, K. Matyjaszewski, I. I. Potemkin, A. R. Khokhlov, and M. Möller, Macromolecules 34, 8354 (2001).

${ }^{18}$ I. I. Potemkin, A. R. Khokhlov, S. Prokhorova, S. S. Sheiko, M. Möller, K. L. Beers, and K. Matyjaszewski, Macromolecules 37, 3918 (2004).

${ }^{19}$ R. Zajac and A. Chakrabarti, J. Chem. Phys. 107, 8637 (1997).
${ }^{20}$ A. Striolo and J. M. Prausnitz, J. Chem. Phys. 114, 8565 (2001).

${ }^{21}$ T. E. Creighton, Proteins: Structures and Molecular Properties, 2nd ed. edited by (W. H. Freeman, New York, 1993).

${ }^{22}$ T. Garel and H. Orland, J. Phys. A 23 L621 (1990).

${ }^{23}$ P. Q. Higgs and H. Orland, J. Chem. Phys. 95, 4506 (1991).

${ }^{24}$ A. Striolo, D. Bratko, J. M. Prausnitz, N. Elvassore, and A. Bertucco, Fluid Phase Equilib. 183-184, 341 (2001).

${ }^{25}$ P. H. Verdier and W. H. Stockmayer, J. Chem. Phys. 36, 227 (1962).

${ }^{26}$ N. Metropolis, A. W. Rosenbluth, M. N. Rosenbluth, A. N. Teller, and E. Teller, J. Chem. Phys. 21, 1087 (1953).

${ }^{27}$ S. Asakura and F. Oosawa, J. Chem. Phys. 22, 1255 (1954).

${ }^{28}$ A. Striolo, C. M. Colina, K. E. Gubbins, N. Elvassore, and L. Lue, Mol. Simul. 30, 437 (2004).

${ }^{29}$ J. J. de Pablo, H. C. Ottinger, and Y. Rabin, AIChE J. 38, 273 (1992).

${ }^{30}$ Y. Zhou, C. K. Hall, and M. Karplus, Phys. Rev. Lett. 77, 2822 (1996).

${ }^{31}$ Y. Zhou, M. Karplus, J. M. Wichert, and C. K. Hall, J. Chem. Phys. 107, 10691 (1997).

${ }^{32}$ S. Damodaran, Interfaces, Protein Films, and Foams (Academic, San Diego, 1990).

${ }^{33}$ A. V. Smith and C. K. Hall, Proteins 44, 344 (2001).

${ }^{34}$ K. Leonhard, J. M. Prausnitz, and C. J. Radke, Phys. Chem. Chem. Phys. 5, 5291 (2003)

${ }^{35}$ R. A. L. Jones and R. W. Richards, Polymers at Surfaces and Interfaces (Cambridge University Press, London, 1999).

${ }^{36}$ A. Striolo and J. M. Prausnitz, J. Chem. Phys. 113, 2927 (2000). 